\title{
Performance Analysis of TDCS Based on Adaptive Threshold Zhou Zidong ${ }^{1, a}$, Chen Zili, ${ }^{1, b}$ \\ ${ }^{1}$ Department of UAV Engineering Ordnance Engineering College Shijiazhuang, China

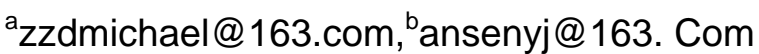

Keywords: TDCS; adaptive threshold; the probability of detection; the probability of false alarm; anti-jamming

\begin{abstract}
The traditional Transform Domain Communication System(TDCS)utilizing the fixed threshold is difficult to adapt to the increasingly complex and variable electromagnetic environment, and the adaptive thresholding methods only concern the probability of detection and ignore the probability of false alarm ,which causes the increase of BER(bit error rate). To tackle with the problems above, a new method of adaptive threshold is proposed. This method combines the probability of detection and the probability of false alarm by setting the weighting factor. The optimal threshold is deduced based on the optimal detection performance and it can also adaptively adjust the threshold value according to the change of channel environment. Theoretical analysis and simulations show that this method can correctly location and eliminate the spectrum of interference. The amplitude spectrum of basic function is generated with almost no interference after interference elimination, which reduces the TDCS's BER and the system's anti-jamming performance is improved.
\end{abstract}

\section{Introduction}

Based on real-time channel evaluation and spectrum sensing, transform domain communication system can dynamically design fundamental modulation waveform to avoid interference in transform domain and transmit signal by threshold decision. TDCS is regarded as a research focus in the field of anti-jamming communication in the future due to its characteristic of active jamming avoidance. Threshold setting is the premise and key steps in TDCS's anti-jamming, especially in the current increasingly complex electromagnetic environment, interference threshold setting is particularly important. Whether the threshold setting is reasonable or not decides the interference elimination and then affects the anti-jamming performance of the system.

In the field of TDCS threshold setting, the following two main aspects have been concerning: adaptive threshold and fixed threshold.

Ref[1-4] set the fixed threshold to $40 \%$ of the maximum of power spectrum value based on simulation experiments. This method is simple to set up, but is clearly not the optimal threshold value for its BER increases because of leaking check and misjudgment. In Ref[5], an algorithm of basis functions amplitude spectrum based on adaptive threshold is proposed. The threshold value can adaptive adjust to the changes of electromagnetic environment. But the proposed method only considers the detection probability while ignoring the false alarm probability, leads to reduction of frequency band utilization. Enlightened by image processing method, a TDCS adaptive threshold setting method based on Otsu is proposed in [6]. The algorithm can maintain well communication performance with strong self-adaptive capacity in high-noise background but is computationally complex.

Aiming at that the fixed threshold is difficult to adapt the diversity of transmission channel and unable to take detection probability and false alarm probability into account, this paper proposed an optimization method of threshold setting which adapts to the change of SNR and reconciles the detection probability and the false alarm probability. Simulations of TDCS show that the BER of the proposed method outperforms the traditional fixed threshold and the algorithm in [5], by $4.15 \mathrm{~dB}$ and 2.77dB. 


\section{Fixed Threshold Setting}

The basis function of transform domain communication system based on fixed threshold is generated as follows.

The TDCS transmitter samples the surrounding electromagnetic environment and estimates the location of interference spectrum by spectrum sensing in transform domain. Then the amplitude spectrum vector without interference is obtained by pre-set threshold decision. If the amplitude value of a certain frequency point exceeds the set threshold, the frequency amplitude value is set to " 0 ", on the other hand , is set to " 1 ". Multiplying the magnitude spectrum vector composed of " 0 " and " 1 " with pseudo-random phase can get the modulation basis functions.

Fixed threshold setting is based on a large number of simulation experiments, not on theoretic analysis and deduction. In [4], for example, setting 30\%, 40\%, 50\% of maximum power magnitude as threshold respectively, simulation results show that the BER performance of $40 \%$ of maximum power magnitude is excellent by comparing BER. Obviously, it is not optimal and the fixed threshold is difficult to adapt to the changing electromagnetic environment.

\section{Adaptive Threshold Setting}

The fixed threshold decision used in traditional TDCS is unable to adjust to the increasingly complex electromagnetic environment. An adaptive threshold algorithm that adapts to the change of SIR and reconcile the the detection probability and the false alarm probability is proposed in this paper.

Interference Signal Detection Model. The binary hypothesis test model of TDCS sampling signal can be expressed as follows.

$$
x(n)=\left\{\begin{array}{c}
H_{0}: w(n) \\
H_{1}: j(n)+w(n)
\end{array}, n=0,1 \ldots N-1\right.
$$

$x(n)$ shows the received sampling signal, $N$ is the number of samples. $j(n)$ is test interference signal with zero mean and variance $\sigma_{j}^{2}$ and $w(n)$ is additive white Gaussian noise with zero mean and variance $\sigma_{n}{ }^{2} \cdot H_{0}$ is the null hypothesis, which indicates the absence of interference, and $H_{1}$ is the alternative hypothesis, which indicates that interference is present.

Energy detection is one of the most commonly used methods in cognitive radio spectrum sensing. The statistic is obtained by sampling the received signals using energy detection method.

$$
E_{x}=\sum_{n=0}^{N-1}|x(n)|^{2}
$$

In [5], the signal detection performance can be described by detection probability $P_{d}$ and false alarm probability $P_{f} . E_{x}$ obeys Gaussain distribution when the number of samples is large enough according to Central Limit Theorem.

$$
\begin{aligned}
& P_{d}=\operatorname{Pr}\left\{E_{x} \geq T \mid H_{1}\right\}=Q\left(\frac{T-\left(\sigma_{j}^{2}+\sigma_{n}^{2}\right)}{\left(\sigma_{j}^{2}+\sigma_{n}^{2}\right)} \bullet \sqrt{\frac{N}{2}}\right) \\
& P_{f}=\operatorname{Pr}\left\{E_{x} \geq T \mid H_{0}\right\}=Q\left(\frac{T-\sigma_{n}^{2}}{\sigma_{n}^{2}} \bullet \sqrt{\frac{N}{2}}\right)
\end{aligned}
$$

$Q(x)$ is Gaussian integral compensation function, and $T$ is decision threshold.

$$
Q(x)=\frac{1}{\sqrt{2 \pi}} \int_{x}^{\infty} e^{-t^{2} / 2} d t
$$

(5) shows that the system detection performance only depends on decision threshold $T$ when noise power $\sigma_{n}^{2}$ and interference signal power ${ }^{2}{ }_{j}^{2}$ are known. If the threshold is set too high and the interference signal power is low, system may not detect the existence of interference signal, which reduces the detection probability and increases the probability of missed detection, and 
mistakenly labeled the spectrum as 1 . The system is modulated with a lot of interference channels, greatly reducing the anti-jamming performance. If the threshold is set too low and the noise power is high, system may judge the background noise as interference signal, which increases the false alarm probability, and labeled the spectrum as 0 , resulting in a waste of frequency spectrum resources. The range of threshold is shown in (6).

$$
\sigma_{n}^{2} \leq T \leq \sigma_{j}^{2}
$$

In order to ensure the anti-jamming performance of TDCS spectrum and reduce the waste of resources, it is necessary to make a further weigh on the detection probability and false alarm probability, thus obtains the optimal threshold decision value and achieves the optimal system detection performance, so as to improve the anti-jamming performance of the system.

The Algorithm of Optimal Decision Threshold. The detection performance $P$ is a function of threshold $T$. Supposed $\alpha, \beta$ is respectively the weighting factors of detection probability $P_{d}$ and false alarm probability $P_{f}$, where $0<\alpha<1,0<\beta<1$ and $\alpha+\beta=1$.Through the above analysis, the greater the probability of detection in theory, the performance of system is better, and the false alarm probability is smaller, better detection performance. So the expression of $P(T)$ is :

$$
P(T)=\alpha P_{d}+\beta\left(1-P_{f}\right)=\alpha Q\left(\frac{T-\left(\sigma_{j}^{2}+\sigma_{n}^{2}\right)}{\left(\sigma_{j}^{2}+\sigma_{n}^{2}\right)} \cdot \sqrt{\frac{N}{2}}\right)+\beta\left[1-Q\left(\frac{T-\sigma_{n}^{2}}{\sigma_{n}^{2}} \cdot \sqrt{\frac{N}{2}}\right)\right]
$$

For convenience, let

$$
\begin{aligned}
& \mathrm{A}=\frac{T-\left(\sigma_{j}^{2}+\sigma_{n}^{2}\right)}{\left(\sigma_{j}^{2}+\sigma_{n}^{2}\right)} \cdot \sqrt{\frac{N}{2}}, \\
& \mathrm{~B}=\frac{T-\sigma_{n}^{2}}{\sigma_{n}^{2}} \cdot \sqrt{\frac{N}{2}}
\end{aligned}
$$

Simplifying $P(T)$ according to the property of $Q(x)$, where $Q(-x)=1-Q(x)$.

$$
P(T)=\alpha Q(\mathrm{~A})+\beta[Q(-\mathrm{B})]=\frac{\alpha}{\sqrt{2 \pi}} \int_{\mathrm{A}}^{\infty} e^{-\frac{t^{2}}{2}} d t+\frac{\beta}{\sqrt{2 \pi}} \int_{-\mathrm{B}}^{\infty} e^{-\frac{t^{2}}{2}} d t
$$

So the threshold optimization problem is transformed into the demand function of the maximum problem, that is calculating the $\max [P(T)]$ under the condition $\sigma_{n}^{2} \leq T \leq \sigma_{j}^{2}$.

If it proves that $P(T)$ is convex function, the problem is transformed into strictly convex optimization problem.

Taking first order derivative of function $P(T)$ can get

$$
\frac{\partial P(T)}{\partial T}=-\frac{\alpha}{\sqrt{2 \pi}} e^{-\frac{\mathrm{A}^{2}}{2}} \frac{\partial(\mathrm{A})}{\partial T}+\frac{\beta}{\sqrt{2 \pi}} e^{-\frac{\mathrm{B}^{2}}{2}} \frac{\partial(\mathrm{B})}{\partial T}
$$

Taking second order derivative of function $P(T)$ can get

$$
\begin{aligned}
\frac{\partial^{2} P(T)}{\partial T^{2}} & =-\frac{\alpha}{\sqrt{2 \pi}}(-\mathrm{A}) e^{-\frac{\mathrm{A}^{2}}{2}} \frac{\partial(\mathrm{A})}{\partial T} \cdot \frac{\partial(\mathrm{A})}{\partial T}+\frac{\beta}{\sqrt{2 \pi}}(-\mathrm{B}) e^{-\frac{\mathrm{B}^{2}}{2}} \frac{\partial(\mathrm{B})}{\partial T} \cdot \frac{\partial(\mathrm{B})}{\partial T} \\
& =\frac{\alpha \mathrm{A}}{\sqrt{2 \pi}}\left[\frac{\partial(\mathrm{A})}{\partial T}\right]^{2} e^{-\frac{\mathrm{A}^{2}}{2}}-\frac{\beta \mathrm{B}}{\sqrt{2 \pi}}\left[\frac{\partial(\mathrm{B})}{\partial T}\right]^{2} e^{-\frac{\mathrm{B}^{2}}{2}}
\end{aligned}
$$

In condition of $\sigma_{n}^{2} \leq T \leq \sigma_{j}^{2}$, we get

$$
\begin{aligned}
& \mathrm{A}=\frac{T-\left(\sigma_{j}^{2}+\sigma_{n}^{2}\right)}{\left(\sigma_{j}^{2}+\sigma_{n}^{2}\right)} \cdot \sqrt{\frac{N}{2}}<0 \\
& \mathrm{~B}=\frac{T-\sigma_{n}^{2}}{\sigma_{n}^{2}} \cdot \sqrt{\frac{N}{2}}>0
\end{aligned}
$$

And $0<\alpha<1,0<\beta<1$, so

$$
\frac{\partial^{2} P(T)}{\partial T^{2}}<0
$$


By the definition of convex function, $P(T)$ is convex function, where $\sigma_{n}{ }^{2} \leq T \leq \sigma_{j}{ }^{2}$, and $\max [P(T)]$ represents strictly convex optimization problem. So choosing the optimal threshold is transformed into solving the partial differential equation $\partial P(T) / \partial T=0$.

$\frac{\partial P(T)}{\partial T}=-\frac{\alpha}{\sqrt{2 \pi}} e^{-\frac{\mathrm{A}^{2}}{2}} \frac{\partial(\mathrm{A})}{\partial T}+\frac{\beta}{\sqrt{2 \pi}} e^{-\frac{\mathrm{B}^{2}}{2}} \frac{\partial(\mathrm{B})}{\partial T}=0$

Simplifying the equation can get:

$$
\mathrm{A}^{2}-\mathrm{B}^{2}=2 \ln \left[\frac{\alpha \sigma_{n}^{2}}{\beta\left(\sigma_{j}^{2}+\sigma_{n}^{2}\right)}\right]
$$

Solving the quadratic equation by formula:

$$
\begin{aligned}
& T_{1,2}=\frac{1 \pm \sqrt{1+\frac{X+Y}{Y-X} \cdot \frac{2}{N} Z}}{\left(\frac{1}{X}+\frac{1}{Y}\right)}=\frac{X Y}{X+Y}\left(1 \pm \sqrt{1+\frac{X+Y}{Y-X} \cdot \frac{2}{N} Z}\right) \\
& \left\{\begin{array}{l}
X=\sigma_{j}^{2}+\sigma_{n}^{2} \\
Y=\sigma_{n}^{2} \\
Z=2 \ln \left[\alpha \sigma_{n}^{2} / \beta\left(\sigma_{j}^{2}+\sigma_{n}^{2}\right)\right]
\end{array}\right.
\end{aligned}
$$

Considering the decision threshold value is actually real number and non-negative. We can get the optimal threshold value by substituting $j n r=\sigma_{j}^{2} / \sigma_{n}^{2}$ into (18).

$$
T=\sigma_{n}^{2} \bullet \frac{1+j n r}{1+2 j n r}\left\{\left[1-\frac{4(1+2 j n r)}{N} \ln \left[\frac{\alpha}{\beta(1+j n r)}\right]\right]\right\}
$$

It can be seen from above that the optimal decision threshold can be calculated by eliminating interference when

\section{Simulation and Analysis}

This paper uses Matlab to construct a simulation platform and analyses the TDCS system based on adaptive threshold. Related simulation parameters are as follows: interference signals using the complex interference signal that coexistence of Multi-tone interference and Narrowband interference, of which the frequency point of Multi-tone interference is set to $75 \mathrm{~Hz}, 150 \mathrm{~Hz}, 200 \mathrm{~Hz}, 300 \mathrm{~Hz}$ and $400 \mathrm{~Hz}$, and the frequency band of Narrowband interference ranges of $300 \sim 400 \mathrm{~Hz}$; using additive white gaussian noise channel with the noise variance set to 1 , that is $\sigma_{n}^{2}=1$; the modulation mode is BPSK; the fixed SNR is $0 \mathrm{~dB}$ and SIR ranges of $0 \sim 15 \mathrm{~dB}$; the number of samples is 512; weighting factors of detection probability and false alarm probability are set to 0.5 , respectively, that is $\alpha=\beta=0.5$.

Fig. 1 simulates the interference power spectrum at -3dB SNR and the threshold value obtained by traditional threshold method (i.e.,40\% of the maximum power spectrum value), and the amplitude spectrum vector of basis function composed of 0 and 1.Fig.2 simulates the amplitude spectrum vector of basis function derived by the proposed adaptive threshold algorithm under the same conditions. Comparing Fig.1 and Fig.2, it is found that the traditional fixed threshold fails to detect the interference in $75 \mathrm{~Hz}, 150 \mathrm{~Hz}$ and $200 \mathrm{~Hz}$, while the proposed threshold setting method which taking detection probability and false alarm probability into consideration can accurately locate interference signal, eliminate interference spectrum, obtain the amplitude spectrum vector of TDCS basis function with non-interference and lay a good foundation of anti-jamming for basis function. 

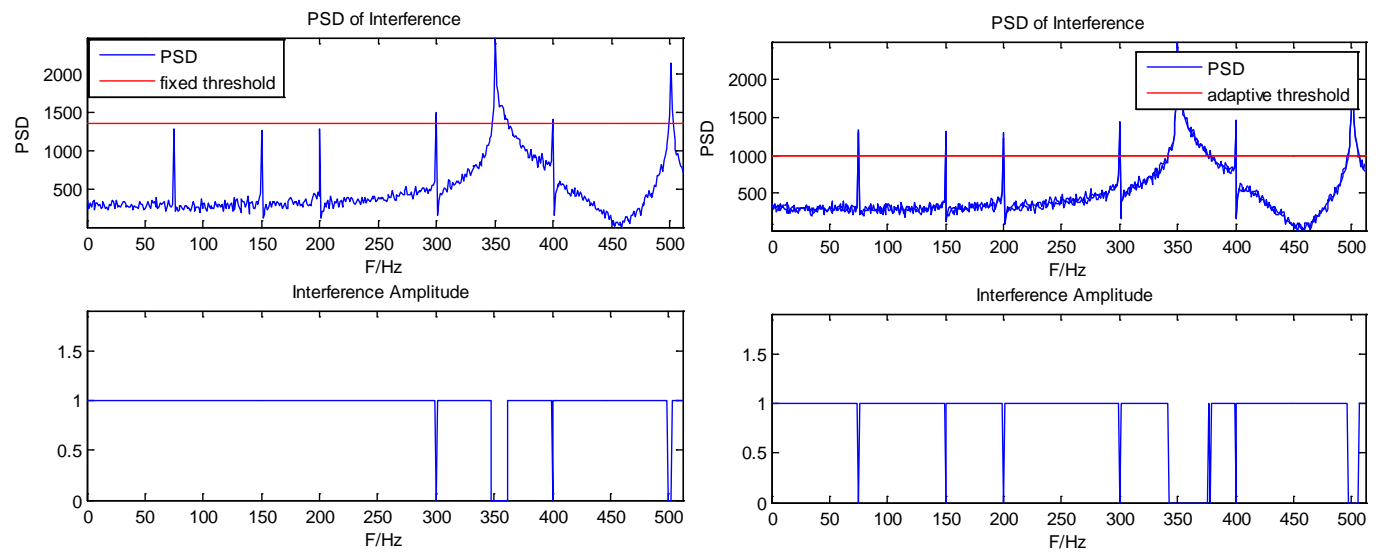

Fig 1.Generation of basic function amplitude

Fig 2.Generation of basic function based on fixed threshold amplitude based on adaptive threshold

To further analysis the advantage of the proposed threshold setting method in adaptive anti-jamming, the simulation of the SNR is fixed and the BER curve of TDCS under different conditions of SIR is shown in figure 3.

Fig.3 shows that the traditional fixed threshold cannot adaptive adjust to the changes of channel environment and prone to leak check and misjudgment, leading to high BER and poor performance of anti-jamming. The threshold setting method proposed in [5] can adaptive adjust the noise and has lower BER compared with the traditional fixed threshold. This paper adopts the method that can reconcile the detection probability and false alarm probability, and can be adaptive to the changes of channel environment, which has lower BER and good anti-jamming performance.

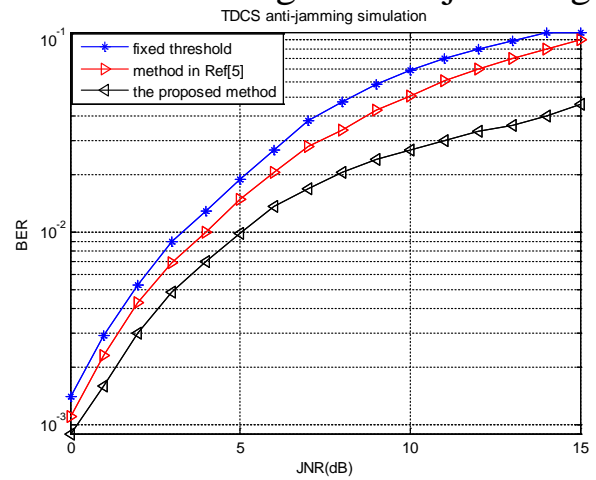

Fig 3.BER performance of TDCS in different threshold methods

For the convenience of comparison the performance of method A with method $\mathrm{B}$, the definition of improvement degree of BER is:

$$
I=10 \log _{10}\left(\frac{B E R_{A}}{B E R_{B}}\right) \quad(d B)
$$

It can be drawn from the figure that the proposed method yields lower BER of TDCS than traditional threshold method and [5] in the order of $4.15 \mathrm{~dB}$ and $2.77 \mathrm{~dB}$ at $10 \mathrm{~dB}$ SIR.

\section{Conclusion}

Aiming at the shortcomings of the traditional fixed threshold, which is prone to leak check and misjudgment, the paper firstly proposes a new setting method of TDCS adaptive threshold, theoretical analyses and derives the optimal threshold expression that takes detection probability and false alarm probability into account. The threshold can adjust different channel environment adaptively. The Matlab simulation experiment proves that TDCS based on this algorithm can accurately locate and eliminate interference, generate amplitude spectrum of basis function without interference, and lay the foundation for improvement of anti-jamming performance. Finally, compared with other two threshold setting methods, further results show that this method can effectively improve the anti-jamming performance of the system. The next step research will be further optimize the design of basis function to achieve non-interference communication. 


\section{References}

[1] E.H. German, Transform Domain Signal Processing Study Final Report[R]. Tech. rep.,Reisterstown, MD: Contract: Air Force F30602-86-C-0133, DTIC: ADB132635, Aug. 1988.

[2] Radcliffe, Rodney A. Design and Simulation of a Transform Domain Communication System, MS Thesis, Air Force Institute of Technology, 1996.

[3] Swackhammer, Patrick J. Design and Simulation of a Multiple Access Transform Domain Communication System, MS Thesis, Air Force Institute of Technology, 1999.

[4] He Zhiqing. Research on the Design and Simulation of Transform Domain Communication System[D], Xi’an:Northwestern Polytechnical University, 2003.

[5] Zeng Wu,Da Xinyu,Shen Yong,et.al. Basis Function Generation Method of TDCS Based on Adaptive Threshold Judgment[J]. Video Engineering, 2011, 35(23):103-105.

[6] Wang Jie,Mao Yuquan,Zhang Hengyang,et.al. TDCS Adaptive Threshold Selection Algorithm Based on Otsu Guidelines[J]. Journal Of Air Force Engineering University(Natural Science Edition),2013.14(4):71-75.

[7] Prashob R Nair, A P Vinod, A K Krishna. An adaptive threshold based energy detector for spectrum sensing in cognitive radios at low SNR[C], 2010 IEEE International Conference on Communication Systems, 2010:574-578.

[8] Prashob R Nair;A. P. Vinod;Anoop Kumar Krishna. An energy detector for cognitive radios in channels at low SNR using adaptive threshold[C], 2011 8th International Conference on Information, Communications \& Signal Processing, 2011:1-5.

[9] Xu Junfeng. The Comparison of Anti-jamming Performance Between TDCS and DSSS[D], Nanjing: Nanjing University of Science and Technology,2007 